REVIEW ARTICLE

\title{
Revisiting the relationship between resistance training dose and strength gains: what is the real role of volume?
}

\author{
Witalo Kassiano, Bruna Costa, Dalton de Lima-Júnior, Petrus Gantois, \\ Fabiano de Souza Fonseca, Leonardo de Sousa Fortes
}

Resistance training (RT) volume is considered a critical variable to induce neuromuscular adaptations (i.e., increased muscular strength). However, emerging findings have allowed us to revisit the role of volume in strength gains.

Objectives: In the present study, we seek to present these emerging findings to discuss the role of RT volume in one-repetition maximum (1RM), isometric, and isokinetic strength gains. In addition, we propose alternative ways to test whether or not volume plays a determining role in strength adaptation.

Design \& methods: We reviewed the literature on RT volume and strength. In addition, we examined the RT literature to provide alternative ways to investigate the effect of volume on changes in strength.

Results/conclusions: From the recent findings, we argue that an increase in strength can be achieved through a refined interaction between skill enhancement, regular use of high loads, and neuromuscular fatigue management; these points can be obtained from different RT volumes. From an inquiring point of view, we suggest that future investigations that aim to verify the effects of volume on muscular strength may consider the inclusion of high load sessions $(80-100 \%$ of $1 \mathrm{RM}$ ), periodically (e.g., every three or four weeks), in both low and high-volume groups, as well as considering the inclusion of different strength measures (e.g., isokinetic and isometric). We believe that this will help to clarify the nature of the relationship between RT volume and strength adaptations.

(Journal of Trainology 2021;10:10-15)

Key words: neuromuscular adaptations $\boldsymbol{\square}$ resistance training $\boldsymbol{\square}$ isometric strength $\boldsymbol{\square}$ isokinetic strength

\section{INTRODUCTION}

Muscular strength is recognized as one of the most important physical attributes of overall health status and athletic performance. ${ }^{1-3}$ The effectiveness of resistance training (RT) to improve muscular strength is well-established..$^{1,4,5}$ In order to increase muscular strength gains through RT, strength coaches can manipulate several training parameters (i.e., rest interval, repetition tempo, intensity, and volume). ${ }^{1}$ Among these RT prescription parameters, training intensity (i.e., \% of one-repetition maximum [1RM]) plays a key role in long-term muscular strength gains. This premise relies on the so-called "specific adaptations to imposed demands" principle. ${ }^{6,7}$ Furthermore, it has been suggested that RT volume is another essential prescription parameter purported to improve muscular strength. ${ }^{1,8,9}$

The most common parameters used to quantify RT volume are the number of sets performed and/or the volume-load (load lifted multiplied by the number of repetitions). ${ }^{10-12}$ In this context, different studies-including systematic reviews with meta-analysis - have suggested a positive dose-response relationship between training volume and the increase in muscular strength. ${ }^{89}$ For example, it was demonstrated that performing higher RT volume ( $>5$ sets/week per exercise) was more effective than low volume $(\leq 5$ sets/week per exercise) to increase 1RM strength. ${ }^{8}$ However, in that study, the dose-response relationship was not confirmed. ${ }^{13}$ Furthermore, emerging findings did not observe differences in 1RM gains between low, moderate, and high RT volume. ${ }^{14-16}$

Additionally, it should be acknowledged that the method used to assess muscular strength may provide controversial findings concerning the effects of RT volume on strength gains. Although the 1RM test is often used to measure muscular strength, it has been suggested that assessing differences between pre- to post-training interventions on 1RM assessment might not represent the broad spectrum of muscular strength adaptations attained through RT.,17 Thus, the assessment of muscular strength in different pattern (e.g., isometric and isokinetic) has been encouraged. ${ }^{6,17}$

Previous studies on RT volume raised some questions about its role and muscular strength adaptations when non-specific muscular strength tests were performed. ${ }^{18-20}$ Therefore, the aims of the present study were: a) critically review the role of RT volume on muscular strength adaptations (1RM, isokinetic, and isometric measures), in light of the evidence support-

Received February 26, 2021; accepted July 6, 2021

From the GEPEMENE-Metabolism, Nutrition and Exercise Laboratory, Physical Education and Sport Center, Londrina State University, Brazil (W.K., B.C.), Department of Physical Education of Federal University of Paraiba, João Pessoa, Brazil (D.L.J., P.G., L.S.F.) and Department of Physical Education of Federal Rural University of Pernambuco, Brazil (F.S.F.)

Communicated by Takashi Abe, Ph.D.

Correspondence to: Witalo Kassiano, Metabolism, Nutrition, and Exercise Laboratory. Physical Education and Sport Center, Londrina State University,

Rodovia Celso Garcia, km 380, 86057-970, Londrina, Brazil

E-mail: acc.witalo@gmail.com

Journal of Trainology 2021;10:10-15 @2012 The Active Aging Research Center http://trainology.org/ 
ing the dose-response relationship and emerging findings, and b) to provide alternative ways to investigate the nature of the relationship between volume and strength adaptations in future studies. The findings might assist strength coaches in designing optimal RT programs aimed at developing longterm muscular strength.

\section{Support for the dose-response relationship between volume and 1RM strength gains}

Several meta-analyses indicate that, to some extent, the higher the RT volume, the more pronounced the strength gains. ${ }^{8,9,13}$ For example, in the Krieger ${ }^{9}$ meta-analytic study, the authors concluded that multiple sets promote greater muscular strength improvements than single-set RT programs. More precisely, Krieger ${ }^{9}$ showed that for muscular strength gains, two and three sets per exercise were more effective than one set. However, four to six sets did not provide additional gains compared to lower volumes ( $\leq 3$ sets). Furthermore, it seems that a greater number of sets does not necessarily translate into higher neural adaptations ${ }^{13,19,21}$. These findings suggest that the enhancement in muscular strength is related to learning effects (i.e., skill improvement) due to repeated exposure to the specific resistance exercise. $^{22,23}$. Furthermore, when more than three sets are performed, it seems that these neural learning adaptation effects might be attenuated (this hypothesis is detailed in section 3), raising some questions about their dose-response relationship.

In another meta-analysis, Borde et al. ${ }^{13}$ investigated the effects of RT volume on strength when considering only studies with older adults. In that study, no dose-response relationship was observed between RT volume and strength gains when performing meta-regression analyses. Likewise, Peterson et al. ${ }^{24}$ did not identify any significant relationships between RT volume and muscular strength gains in older adults. Recently, Ralston et al. ${ }^{8}$ analyzed the effects of the weekly-sets volume on 1RM strength gains in adult men and found that performing more than five sets per week (moderate volume) induced significant, although trivial (effect size $=$ 0.18), additional gains compared to low RT volume. In that meta-analysis, beyond the trivial advantage of performing five or more sets per week, the authors draw attention to the possibility that the greater effectiveness of moderate-high volume protocols may not have been solely related to exercise volume but could have been driven by the greater exposure to exercise with characteristics similar to the 1RM test. This statement is supported by the lack of difference in strength gains between different RT sets-volumes, when strength was measured in a non-specific way (e.g., isometric and isokinetic strength). ${ }^{19,20,25}$ Furthermore, emerging findings show similar gains in muscular strength in response to low, moderate, and high-volume protocols. ${ }^{14-16,26}$

\section{Emerging findings and argumentation}

The number of studies investigating RT volume in muscular adaptations has been growing in several populations and in various training ages. ${ }^{27,28}$ These studies enable strength and conditioning scientists and coaches to improve their under- standing of the dose-response relationship, or the lack of, between RT volume and muscular strength adaptations. Recently, it has been indicated that strength gains may not differ amongst RT volumes (e.g., one vs. three vs. five sets), suggesting that optimal strength increases could occur in alternative ways rather than through the increase in RT volume. ${ }^{15,29}$ Previous studies on RT volume in older women ${ }^{14}$, resistance-trained $\operatorname{men}^{16}$, and powerlifters ${ }^{15}$ showed similar results amongst different RT volumes. Overall, this current body of knowledge might be used to guide strength coaches to manage their decisions from an evidence-based approach to improve the effectiveness of their RT prescription. Given the scenario described above, we detail and discuss the results of studies investigating the effect of volume (number of sets and volume-load) on strength adaptations.

\section{Number of sets and 1RM strength}

The number of sets is the most common parameter to quantify RT volume. ${ }^{28}$ In this context, some studies have observed similar 1RM strength gains with low, moderate, and high set volumes (e.g., 1, 3, and 5 sets, respectively). ${ }^{14-16,26}$ For instance, similar improvements in muscular strength were found following 12-weeks of RT in older women performing one (upper-limb $=37.1 \%$, lower-limb $=16.3 \%$ ) or three sets (upper-limb $=27.4 \%$, lower-limb $=21.7 \%$ ) per exercise (volume-load $=40.9$ vs. 105.8 ton, respectively). ${ }^{14}$ Resistancetrained adults, a population that would theoretically benefit more from a larger training volume, demonstrated similar muscular strength gains when training with one (upper-limb = $9.3 \%$, lower-limb $=18.9 \%$ ), three (upper-limb $=5.7 \%$, lower$\operatorname{limb}=13.6 \%$ ), and five sets (upper-limb $=6.8 \%$, lower-limb $=$ $19.6 \%) .{ }^{16}$ More recently, Androulakis-Korakakis et al. ${ }^{15}$ demonstrated, in a pilot study, that performing single-set, singlerep, RPE-based training may be an effective strategy for some beginner-intermediate powerlifters to increase maximum strength similarly to high-volume periodized training. ${ }^{15}$ Therefore, similar muscular strength gains in different populations and training ages suggest that the RT volume may not determine the magnitude of strength adaptations.

In scientific investigations, maximum muscular strength is usually measured through the $1 \mathrm{RM}$ test. An increasing number of studies point out that, perhaps, improvements in 1RM test performance resemble a skill, which is enhanced when the training characteristics, such as muscle action, velocity, range of motion, and external load (\%RM) are similar to those used in the test. ${ }^{22,23,25}$ For example, Dankel et al. ${ }^{23}$ evaluated the effect of 21 consecutive sessions of the 1RM familiarization test compared to traditional training on strength gains, through an intra-subject design study. The results showed that the arm that performed only the 1RM test in all sessions increased the load lifted similarly when compared to the arm that performed three sets at $70 \% 1 \mathrm{RM}$ throughout the experiment (1.9 kg vs. $2.2 \mathrm{~kg}$, respectively). Likewise, Mattocks et al. ${ }^{25}$ observed that the $1 \mathrm{RM}$ test practice promoted similar strength gains to a multiple sets scheme of 8-12RM.

Previous studies using low RT volume but repeated expo- 
sure to the 1RM test during RT programs support the hypothesis of a learning effect on 1RM gains rather than a doseresponse relationship between them. ${ }^{5,30,31}$ In a comprehensive study by Bickel et al. ${ }^{31}$, young and older adults performed an RT program divided into two phases with the following characteristics: in the first phase, the subjects trained three times a week, over 16 weeks. The sessions were composed of three sets in the knee extension, leg press, and squat; in the second phase, which extended from weeks 17 to 48, the subjects were randomly divided into three groups: (1) detraining, reducing the volume to (2) one-third and (3) one-ninth of the volume performed in the first phase. The 1RM test was performed for the knee extension every four weeks for 32 weeks, and after this, in weeks 40 and 48. Interestingly, after the end of phase two, the 1RM performance in the groups that reduced to onethird and one-ninth of the initial volume was significantly greater than the baseline and week 16; suggesting that the regular practice of exercise with characteristics similar or identical to the 1RM test seems to be sufficient to increase performance, even when training with low RT volume (i.e., 1/3 or $1 / 9$ of initial RT volume). Together, these findings indicate that increased 1RM strength can be better explained by skill improvement - through increased neural drive of agonist muscles, adaptations of motor neuron properties, and reduced co-activation of antagonists. ${ }^{32,33}$ In this regard, high loads ( $\geq 70 \% 1 \mathrm{RM}$ ) and not greater RT volume $\mathrm{R}^{7,34,35}$ might optimize strength adaptations.

\section{Volume-load and 1RM strength}

The volume-load, obtained by multiplying the number of repetitions and load, is another way to quantify the RT volume. ${ }^{1}$ Concerning this metric and its effects on the increase in strength, the dose-response relationship appears to be even more trivial. First, the volume-load is influenced by the relative intensity (i.e., \%RM). For example, a high number of repetitions with low-load $(\leq 60 \% 1 \mathrm{RM})$ can result in a high volume-load. However, it generally induces similar or smaller increases in strength compared to other protocols, including a low number of repetitions and high-load ( $>60 \% 1 \mathrm{RM})$, resulting in a lower volume-load. ${ }^{28,36}$ Second, even in studies with equalized relative intensity, greater volume-load-due to a greater number of repetitions-does not induce additional muscle strength gains, regardless of the sample characteristics (i.e., physically active, strength-trained, or athlete subjects). ${ }^{37-39}$

An increasing body of knowledge using the velocity-based training (VBT) approach (i.e., including different velocity loss thresholds to determine set volume) has accumulated evidence to support the statement that RT volume-load does not play a determinant role in muscular strength adaptation. For instance, Pareja-Blanco et al. ${ }^{40}$ observed that performing twice as many repetitions ( 143.6 vs. $\sim 305.3)$ - and consequently higher volume-load - with equated relative intensity (70-85\% 1RM) did not promote additional gains in lower limb maximum strength (1RM). In another study, Dorrell et al. ${ }^{39}$ compared the effects of the prescription using the VBT paradigm versus the traditional percentage-based approach. The
VBT group interrupted the sets when the subjects presented a $20 \%$ reduction in mean concentric velocity, while the percentage-based group performed the previously determined number of repetitions. After six weeks of RT, the VBT group demonstrated more significant gains in maximum strength in the bench press, and only this group increased maximum strength in the deadlift exercise. Interestingly, the VBT group significantly performed a lower total volume-load than the percentage-based group. ${ }^{39}$ Similarly, Sánchez-Moreno et al. ${ }^{41}$ reported that interrupting the pull-up exercise when reaching a $25 \%$ reduction $(\sim 50 \%$ in the number of maximum repetitions) in velocity increased the maximum strength, while performing the exercise up to a $50 \%(\sim 85 \%$ in the number of maximum repetitions) velocity reduction did not promote significant increases. Together, these studies suggest that maximum dynamic strength development is obtained regardless of the volume-load. Moreover, this adaptation, at least in part, might be associated with the management of fatigue. ${ }^{42}$

\section{If the volume is not a determinant, how can we explain previous dose-response findings?}

Overall, these emerging findings suggest that high-volume does not seem to be necessary to promote greater gains in maximum strength. However, there is still a question to be answered: What would explain the results of previous studies-including meta-analyses - which suggest a doseresponse relationship? A plausible explanation for the advantage observed for high-volume groups may be the motor task improvement - that occurs, in part, through neural adaptations such as increased neural drive to the agonists and reduced presynaptic inhibition. ${ }^{32-34}$ Participants who perform high-volume are more frequently exposed to exercise than the low RT volume group, which could contribute, in part, to improving performance in the strength test at the end of the study. This hypothesis corroborates a recent review by Spitz et al. ${ }^{22}$. In this review, the authors included studies that compared high and low loads to illustrate the effect of exercise on improving performance in the strength test. Spitz et al. ${ }^{22}$ observed that participants increased maximum strength when frequently exposed to the practice of the 1RM test (e.g., every four weeks), even when training with low loads $(\leq 40 \%$ 1RM).

Returning to the RT volume, if the relative intensity $(\% \mathrm{RM})$ remains the same, the subjects who perform more sets are exposed more often to the practice of a particular exercise, and this difference can favor improvement in performance in the 1RM test. On the other hand, if the subjects in the low RT volume group are exposed periodically to the execution of a motor task with characteristics similar to the 1RM test-with similar muscle action, velocity, range of motion, and $\% \mathrm{RM}-$, it is possible to assume that the differences between low and high volume in improving the 1RM are mitigated. However, this hypothesis warrants further investigation. If confirmed, this assumption opens up other research questions, such as; is there an optimal RT frequency of sets and sessions with similar characteristics to the 1RM test? What is the optimal dose of high load sets to promote optimal 
1RM strength? Despite lacking direct evidence, this hypothesis (i.e., effects of the number of times/sets that individuals are exposed to exercise) is supported indirectly by the similarity between the effects of low and high RT volume on strength changes when strength is measured in a non-specific way, as discussed in the next section.

\section{The role of volume on isometric and isokinetic strength}

Importantly, we need to describe what pattern of muscular strength we are evaluating since this phenomenon can be analyzed differently. ${ }^{17,43}$ Specifically, muscular strength, defined as the muscle ability to generate force against external resistance, is a multifactorial ability measured using several methods (e.g., voluntary, isotonic, isometric). The development of this construct depends on the combination of different morphological (e.g., muscle architecture) and neural factors (e.g., increased central motor drive, elevated motoneuron excitability, reduced presynaptic inhibition, and adaptations in properties of the motor neurons). ${ }^{32,33,44}$ Concerning the influence of RT volume on muscular strength, the divergence between findings in the scientific literature has intensified discussions about the existence of a relationship between RT volume and muscular strength gains measured in a non-specific way. ${ }^{19,25,45-47}$

Investigations conducted by different research groups have pointed out that the volume has a low impact on the magnitude of isometric and isokinetic strength changes. ${ }^{19,25,47}$ For example, Radaelli et al. ${ }^{19}$ investigated the effect of RT volume manipulation (one vs. three sets) in older women and observed a higher increase in the 1RM performance of the knee extension for the three set group compared to the one set group after 20-weeks of RT; however, there were no differences in isometric strength gains between groups. Similarly, Mitchell et al. ${ }^{47}$, when comparing one set at $80 \% 1 \mathrm{RM}$ vs. three sets at $80 \% 1 \mathrm{RM}$ vs. three sets at $30 \% 1 \mathrm{RM}$, verified an effect for training intensity but not for volume in the 1RM test. Moreover, when evaluating different strength patterns, such as the rate of force development, maximal voluntary contraction force, and knee extension maximal power output, the magnitude of the adaptations was smaller for low intensities, and no difference was observed between different RT volumes.

In another investigation, Radaelli et al. ${ }^{18}$ observed that the strength adaptations were similar in both the 1RM test and isometric strength when training with one and three sets in older women. In a more comprehensive investigation, the researchers evaluated, in addition to isotonic strength (i.e., $1 \mathrm{RM}$ ), the isometric and isokinetic torque of adult men. ${ }^{25}$ The experiment consisted of one group that performed the 1RM test and another group that performed four sets ( $\sim 8 \mathrm{RM}-12 \mathrm{RM})$ for eight weeks. ${ }^{25}$ Interestingly, both groups presented similar increases in $1 \mathrm{RM}$, isometric, and isokinetic torque. ${ }^{25}$ Taken together, these findings illustrate that when measured in a non-specific way, the possible advantages in strength gains from performing a greater volume are attenuated or absent; nonetheless, these findings are not universal. ${ }^{46}$

The lack of differences in non-specific strength gains when performing different RT volumes might be related to similar neural adaptations in response to low and high RT volume. ${ }^{19,21,25}$ For example, Marshall et al. ${ }^{21}$ did not observe differences in quadriceps electromyography activation and isometric strength between one, four, and eight sets in resistance-trained males after 10-weeks of RT. Likewise, Radaelli et al. ${ }^{19}$ did not observe differences in the muscular activation of the quadriceps and biceps brachii and isometric strength in response to 20 -weeks of one and three sets in older women. This indicates that the similarity of isometric and isokinetic strength adaptations between low and high RT volume may, in part, be due to similarities in neural adaptations in the agonist muscles, at least concerning peripheral adjustments (e.g., surface electromyography); despite this, we have not ruled out possible effects of volume on changes in the motor cortex and supraspinal levels.

Additionally, the greater repeated exposure might improve the skill/performance in the 1RM test through adjustments at the central and peripheral levels specific to the pattern of muscle action, repetition tempo, range of motion, and muscle groups. ${ }^{1,48}$ This is important because there is a dissimilarity of neural activity patterns between isotonic, isokinetic, and isometric tasks - such as unique spatial and spectro-temporal electrocortical signatures, recruitment of the components of the muscular groups ${ }^{48-51}$. Together with the limited doseresponse relationship between RT volume and the magnitude of neural adaptations, this aspect might partially explain why higher isotonic RT volumes do not culminate in greater nonspecific strength adaptations (e.g., isometric and isokinetic).

\section{PERSPECTIVES}

From the arguments presented above, we propose that if the goal is to increase maximum strength (i.e., 1RM), this target can be achieved through a refined interaction between improved skill, periodic use of high load, and management of neuromuscular fatigue. In practical terms, these three points can be achieved with low to moderate RT volume programs, as long as the exercise is performed with high effort. Importantly, we do not dismiss the RT volume as playing a significant role in strength adaptations; although this role appears to be less than previously thought, higher RT volume remains an effective alternative to optimize strength gains in subjects who cannot tolerate training with high loads. Furthermore, concerning the different measurement patterns of strength (e.g., isokinetic and isometric), the RT volume does not seem to affect the magnitude of the changes.

From an inquiring point of view, we suggest that future investigations aim to verify the effects of volume on muscular strength increase, considering the inclusion of high load sessions $(80-100 \%$ of $1 \mathrm{RM})$, periodically (e.g., every three or four weeks), in both low and high-volume groups. This will enable verification of whether higher volume has additional effects on the 1RM strength gains. In addition, we emphasize the need for studies that apply different training volumes and evaluate different patterns of muscular strength to capture a better image of kinetic adaptations in response to different doses of RT. Otherwise, the extrapolations are restricted, in 
the case of $1 \mathrm{RM}$, to a single strength measure. Thus, investigations that evaluate different parts of the strength spectrum will have an even greater appeal since the answers to the question "does high volume correspond to greater strength adaptations?" are of interest to diverse populations, regardless of chronological age or training status.

\section{Compliance with Ethical Standards}

Funding No external sources of funding were used in the preparation of this article.

Conflicts of interest Witalo Kassiano, Bruna Daniella de Vasconcelos Costa, Dalton de Lima-Júnior, Petrus Gantois, Fabiano de Souza Fonseca, and Leonardo de Sousa Fortes declare that they have no conflicts of interest that are relevant to the content of this article.

\section{REFERENCES}

1. ACSM. American College of Sports Medicine position stand. Progression models in resistance training for healthy adults. Med Sci Sports Exerc 2009;41:687-708.

2. Suchomel TJ, Nimphius S, Stone MH. The importance of muscular strength in athletic performance. Sports Med 2016;46:1419-1449.

3. Ruiz JR, Sui X, Lobelo F et al. Association between muscular strength and mortality in men: prospective cohort study. BMJ 2008;337:a439.

4. Kassiano W, Costa BDV, Nunes JP et al. Does resistance training promote enough strength increases to move weak older women to better strength categories? Exp Gerontol 2021; Online ahead of print.

5. Antunes M, Kassiano W, Silva AM et al. Volume reduction: which dose is sufficient to retain resistance training adaptations in older women? Int $J$ Sports Med 2021; in press.

6. Buckner SL, Kuehne TE, Yitzchaki N et al. The generality of strength adaptation. J Trainol 2019;8:5-8.

7. Nunes JP, Ribeiro AS, Schoenfeld BJ et al. Comment on: "comparison of periodized and non-periodized resistance training on maximal strength: a meta-analysis". Sports Med 2018;48:491-494.

8. Ralston GW, Kilgore L, Wyatt FB et al. The effect of weekly set volume on strength gain: a meta-analysis. Sports Med 2017;47:2585-2601.

9. Krieger JW. Single versus multiple sets of resistance exercise: a metaregression. J Strength Cond Res 2009; 23: 1890-1901.

10. Baz-Valle E, Fontes-Villalba M, Santos-Concejero J. Total number of sets as a training volume quantification method for muscle hypertrophy: a systematic review. J Strength Cond Res 2021;35:870-878.

11. Scott BR, Duthie GM, Thorrnton HR et al. Training monitoring for resistance exercise: theory and applications. Sports Med 2016;46:687-698.

12. Schoenfeld BJ, Grgic J, Krieger J. How many times per week should a muscle be trained to maximize muscle hypertrophy? A systematic review and meta-analysis of studies examining the effects of resistance training frequency. J Sports Sci 2019;37:1286-1295.

13. Borde R, Hortobagyi T, Granacher U. Dose-response relationships of resistance training in healthy old adults: a systematic review and metaanalysis. Sports Med 2015;45:1693-1720.

14. Cunha PM, Nunes JP, Tomeleri CM et al. Resistance training performed with single and multiple sets induces similar improvements in muscular strength, muscle mass, muscle quality, and IGF-1 in older women: a randomized controlled trial. J Strength Cond Res 2020;34:1008-1016.

15. Androulakis-Korakakis P, Fisher JP, Kolokotronis P et al. Reduced volume 'daily max' training compared to higher volume periodized training in powerlifters preparing for competition-a pilot study. Sports (Basel) 2018; $6: 86$.

16. Schoenfeld BJ, Contreras B, Krieger J et al. Resistance training volume enhances muscle hypertrophy but not strength in trained men. Med Sci Sports Exerc 2019;51:94-103.

17. Buckner SL, Jessee MB, Mattocks KT et al. Determining strength: a case for multiple methods of measurement. Sports Med 2017;47:193-195.

18. Radaelli R, Botton CE, Wilhelm EN et al. Low- and high-volume strength training induces similar neuromuscular improvements in muscle quality in elderly women. Exp Gerontol 2013;48:710-716.

19. Radaelli R, Botton CE, Wilhelm EN et al. Time course of low- and highvolume strength training on neuromuscular adaptations and muscle quality in older women. Age (Dordr) 2014;36:881-892.

20. Starkey DB, Pollock ML, Ishida Y et al. Effect of resistance training volume on strength and muscle thickness. Med Sci Sports Exerc 1996;28: 1311-1320.

21. Marshall PW, McEwen M, Robbins DW. Strength and neuromuscular adaptation following one, four, and eight sets of high intensity resistance exercise in trained males. Eur J Appl Physiol 2011;111:3007-3016.

22. Spitz RW, Bell ZW, Wong V et al. Strength testing or strength training: considerations for future research. Physiol Meas 2020;41:09TR01.

23. Dankel SJ, Counts BR, Barnett BE et al. Muscle adaptations following 21 consecutive days of strength test familiarization compared with traditional training. Muscle Nerve 2017;56:307-314.

24. Peterson MD, Rhea MR, Sen A et al. Resistance exercise for muscular strength in older adults: a meta-analysis. Ageing Res Rev 2010;9:226-237.

25. Mattocks KT, Buckner SL, Jessee MB et al. Practicing the test produces strength equivalent to higher volume training. Med Sci Sports Exerc 2017; 49:1945-1954.

26. Oliveira-Júnior GN, de Sousa JFR, Carneiro MAS et al. Resistance training volume enhances muscle hypertrophy, but not strength in postmenopausal women: a randomized controlled trial. $J$ Strength Cond Res 2020; Online ahead of print.

27. Figueiredo VC, De Salles BF, Trajano GS. Volume for muscle hypertrophy and health outcomes: the most effective variable in resistance training. Sports Med 2017;48:499-505.

28. Nunes JP, Kassiano W, Costa BDV et al. Equating resistance-training volume between programs focused on muscle hypertrophy. Sports Med 2021;51:1171-1178.

29. Androulakis-Korakakis P, Fisher JP, Steele J. The minimum effective training dose required to increase $1 \mathrm{RM}$ strength in resistance-trained men: a systematic review and meta-analysis. Sports Med 2020;50:751-765.

30. Walker S, Serrano J, Van Roie E. Maximum dynamic lower-limb strength was maintained During 24-week reduced training frequency in previously sedentary older women. J Strength Cond Res 2018;32:1063-1071.

31. Bickel CS, Cross JM, Bamman MM. Exercise dosing to retain resistance training adaptations in young and older adults. Med Sci Sports Exerc 2011; 43:1177-1187.

32. Aagaard P, Simonsen EB, Andersen JL et al. Neural adaptation to resistance training: changes in evoked $\mathrm{V}$-wave and H-reflex responses. $J$ Appl Physiol 2002;92:2309-2318.

33. Del Vecchio A, Casolo A, Negro F et al. The increase in muscle force after 4 weeks of strength training is mediated by adaptations in motor unit recruitment and rate coding. J Physiol 2019;597:1873-1887.

34. Fisher J, Steele J, Smith D. High- and low-load resistance training: interpretation and practical application of current research findings. Sports Med 2017;47:393-400.

35. Morton RW, Colenso-Semple L, Phillips SM. Training for strength and hypertrophy: an evidence-based approach. Curr Opin Physiol 2019;10:9095.

36. Schoenfeld BJ, Grgic J, Ogborn D et al. Strength and hypertrophy adaptations between low- vs. high-load resistance training: a systematic review and meta-analysis. $J$ Strength Cond Res 2017;31:3508-3523.

37. Galiano C, Pareja-Blanco F, Hidalgo de Mora J et al. Low-velocity loss induces similar strength gains to moderate-velocity loss during resistance training. J Strength Cond Res 2020; Online ahead of print.

38. Pareja-Blanco F, Sánchez-Medina L, Suárez-Arrones L et al. Effects of velocity loss during resistance training on performance in professional soccer players. Int J Sports Physiol Perform 2017;12:512-519.

39. Dorrell HF, Smith MF, Gee TI. Comparison of velocity-based and traditional percentage-based loading methods on maximal strength and 
power adaptations. J Strength Cond Res 2020;34:46-53.

40. Pareja-Blanco F, Alcazar J, Sánchez-Valdepeñas J et al. Velocity loss as a critical variable determining the adaptations to strength training. Med Sci Sports Exerc 2020;52:1752-1762.

41. Sánchez-Moreno M, Cornejo-Daza PJ, González-Badillo JJ et al. Effects of velocity loss during body mass prone-grip pull-up training on strength and endurance performance. $J$ Strength Cond Res 2020;34:911-917.

42. Davies $T$, Orr R, Halaki $M$ et al. Effect of training leading to repetition failure on muscular strength: a systematic review and meta-analysis. Sports Med 2016;46:487-502.

43. Nunes JP, Cunha PM, Antunes M et al. The generality of strength: relationship between different measures of muscular strength in older women. Int J Exerc Sci 2020;13:1638-1649.

44. Aagaard P, Andersen JL, Dyhre-Poulsen P et al. A mechanism for increased contractile strength of human pennate muscle in response to strength training: changes in muscle architecture. J Physiol 2001;534:613623.

45. Paulsen G, Myklestad D, Raastad T. The influence of volume of exercise on early adaptations to strength training. J Strength Cond Res 2003;17:
115-120.

46. Rønnestad BR, Egeland W, Kvamme NH et al. Dissimilar effects of oneand three-set strength training on strength and muscle mass gains in upper and lower body in untrained subjects. J Strength Cond Res 2007;21:157163.

47. Mitchell CJ, Churchward-Venne TA, West DW et al. Resistance exercise load does not determine training-mediated hypertrophic gains in young men. J Appl Physiol 2012;113:71-77.

48. Aagaard P, Bojsen-Møller J, Lundbye-Jensen J. Assessment of neuroplasticity with strength training. Exerc Sport Sci Rev 2020;48:151162.

49. Gwin JT, Ferris DP. An EEG-based study of discrete isometric and isotonic human lower limb muscle contractions. J Neuroeng Rehabil 2012;9:35.

50. Pincivero DM, Gandhi V, Timmons MK et al. Quadriceps femoris electromyogram during concentric, isometric and eccentric phases of fatiguing dynamic knee extensions. J Biomech 2006;39:246-254.

51. Babault N, Pousson M, Ballay Y et al. Activation of human quadriceps femoris during isometric, concentric, and eccentric contractions. $J$ Appl Physiol 2001;91:2628-2634. 